\title{
Polarized Classical Non-associative Lambek Calculus and Formal Semantics
}

\author{
Arno Bastenhof \\ Utrecht University
}

\begin{abstract}
While initially motivated for studying natural language syntax, the intuitionistic bias underlying traditional Lambek calculi renders them particularly suitable to a Montagovian formal semantics through the Curry-Howard correspondence. Several recent proposals, however, have departed from the intuitionistic tradition, seeking instead to formulate 'classical' Lambek calculi. We show that this classical turn need not come at the cost of the tight connection with formal semantics, concentrating on De Groote and Lamarche's Classical Non-Associative Lambek calculus (CNL). Our work is founded in Girard's and Andreoli's research into polarities and focused proofs, suggesting the definition of polarized CNL, its connection to De Groote and Lamarche's original proposal explicated through the use of phase spaces. We conclude with a discussion of related literature, particularly Moortgat's Lambek-Grishin calculus.
\end{abstract}

\section{Introduction}

Categorial grammars in the Lambek tradition seek a proof-theoretic explanation of natural language syntax: syntactic categories are formulas and derivations are proofs. Typically, one observes an intuitionistic bias towards single conclusion sequents, the existence of a Montagovian formal semantics thereby being immediate through the Curry-Howard correspondence. Recent years, however, have seen proposals for 'classical Lambek calculi', retaining the resource sensitivity of the traditional systems, while dispensing with their intuitionistic asymmetry in favor of an involutive negation. This article proposes a formal semantics for such systems, concentrating on Classical Non-Associative Lambek calculus (CNL, [7]), arguably the most resource sensitive among its kin.

We proceed as follows. $\S 2$ briefly recapitulates the basics of CNL. In $\S 3$, we define polarized $\mathbf{C N L}\left(\mathbf{C N L}^{\text {pol }}\right)$, drawing from Girard's research into polarities and Andreoli's work on focusing ([5, [1]). Roughly, a dichotomy between positive and negative formulas is uncovered, serving as a guide to Cut-free proof search in avoiding 'don't care' non-determinism. A Montagovian formal semantics is defined for $\mathbf{C N L}^{\text {pol }}$, and briefly motivated through simple analyses of (subject) relativization and quantifier scope ambiguities. We ensure, in $\S 4$, that provability in CNL and $\mathbf{C N L}^{\text {pol }}$ coincide, using an argument involving phase spaces. $\S 5$ concludes with a discussion of several related topics ${ }^{1}$

${ }^{1}$ Throughout this article, in referring to a previously stated definition (lemma, theorem, corollary, figure) $n$, we often use the abbreviation D.n (L.n, T.n, C.n, F.n). 


$$
\begin{array}{ccc}
\frac{\Gamma, \Delta \vdash}{A, A^{\perp} \vdash} A x & \frac{\Gamma \bullet \Delta, \Theta \vdash}{\Delta, \Gamma \vdash} d p^{1} & \frac{\Gamma p^{2}}{\Gamma, \Delta \bullet \Theta \vdash} \\
\frac{\Delta, A \vdash \quad \Gamma, A^{\perp} \vdash}{\Gamma, \Delta \vdash} \text { Cut } & \frac{\Gamma, A \bullet B \vdash}{\Gamma, A \otimes B \vdash} \otimes & \frac{\Gamma, A \vdash \Delta, B \vdash}{\Delta \bullet \Gamma, A \oplus B \vdash} \oplus
\end{array}
$$

Fig. 1. CNL defined. Double inference lines indicate applicability in both directions.

\section{Classical Non-associative Lambek Calculus}

We briefly recapitulate De Groote and Lamarche's sequent calculus for CNL, permitting ourselves a few deviations in notation as motivated below.

Definition 1. Formulas in CNL are generated from positive and negative atoms $p, \bar{p}$ using the multiplicative disjunction $\oplus($ par $)$ and -conjunction $\otimes($ tensor $)$ :

$$
A, B::=p|\bar{p}|(A \otimes B) \mid(A \oplus B)
$$

Classicality is affirmed through linear negation $\cdot{ }^{\perp}$ :

$$
\begin{aligned}
p^{\perp} & =\bar{p} & \bar{p}^{\perp} & =p \\
(A \otimes B)^{\perp} & =B^{\perp} \oplus A^{\perp} & (A \oplus B)^{\perp} & =B^{\perp} \otimes A^{\perp}
\end{aligned}
$$

Definition 2. Structures $\Gamma, \Delta$ combine formulas into binary-branching trees:

$$
\Gamma, \Delta::=A \mid(\Gamma \bullet \Delta)
$$

Definition 3. Figure 1 defines the derivability judgement $\Gamma, \Delta \vdash$, pairing structures $\Gamma, \Delta$ into a left-sided sequent. The rules $\left(d p^{2}\right)$ and $\left(d p^{1}\right)$ remind of both Yetter's cyclicity ([17]), as well als Belnap's display postulates ([2]).

While De Groote and Lamarche employ the more common right-sided sequents, the left-sided notation facilitates a more transparent correspondence with the intuitionistic sequents used in Montagovian semantics, as shown in §3.2.

\section{Polarized CNL}

The current section develops a variation on CNL permitting a correspondence between Cut-free derivations and linear $\lambda$-terms in long $\beta$-normal form. Linguistic applications focus on the relation to a Montagovian-style formal semantics.

\subsection{Motivation}

Our revision of CNL is founded in the research on polarities in logic, initiated in the early nineties through the independent works of Andreoli ([1]) and Girard ([5]). The former's efforts concerned the elimination of inessential non-determinism in naïve backward chaining proof search, while Girard instead 
sought a constructive reading of classical proofs, bypassing Lafont's critical pairs. Both ultimately settled on modified sequent calculi carrying explicit partitionings of formulas correlated with proof-theortic behavior. Downplayed to a strictly multiplicative setting, formulas are of either positive or negative polarity, depending on whether or not their inferences are always invertible, preserving the provability of their conclusion inside their premises. Top-down proof-search then prioritizes the application of invertible inferences, while enforcing maximal chains of non-invertible inferences applied to subformulas of the same initial formula.

Girard's constructivization of classical logic inspired a novel intuitionistic translation, developed further in [16, 18. Roughly, the introduction of double negations is made contingent upon the polarity of the formula being translated, thus achieving parsimony. We adapt Girard's translation to CNL by developing a polarized variant thereof, explicitly distinguishing between positive and negative formulas. Through a Curry-Howard mapping, we obtain a compositional semantics along the lines of [11, illustrated by a few simple case analyses.

\subsection{Polarization and derivational semantics}

Definition 4. Formulas in polarized $\mathbf{C N L}$ (henceforth $\mathbf{C N L}^{\text {pol }}$ ) are inherently positive or negative, with shifts $\uparrow, \downarrow([\underline{6}, \S 5.3)$ establishing communication:

$$
\begin{gathered}
P, Q::=p|(P \otimes Q)|(\downarrow N) \\
M, N::=\bar{p}|(M \oplus N)|(\uparrow P)
\end{gathered}
$$

Linear negation ${ }^{\perp}$ is revised accordingly, satisfying $P^{\perp \perp}=P$ and $N^{\perp \perp}=N$ :

$$
\begin{aligned}
p^{\perp} & =\bar{p} & \bar{p}^{\perp} & =p \\
(P \otimes Q)^{\perp} & =Q^{\perp} \oplus P^{\perp} & (M \oplus N)^{\perp} & =N^{\perp} \otimes M^{\perp} \\
(\downarrow N)^{\perp} & =\uparrow N^{\perp} & (\uparrow P)^{\perp} & =\downarrow P^{\perp}
\end{aligned}
$$

Remark 5. In practice, we assume $\uparrow, \downarrow$ to bind more strongly than $\otimes, \oplus$, and drop brackets accordingly. Thus, $P \otimes \uparrow Q^{\perp}$ abbreviates $\left(P \otimes\left(\uparrow Q^{\perp}\right)\right)$.

The linguistic explanation of Lambek calculi emphasizes a reading of formulas as syntactic categories. Their inductive structure facilitates a tight correspondence with semantic types, the formulas of an intuitionistic calculus used to describe the range of possible denotations for linguistic expressions.

Definition 6. For the purpose of describing the semantic operations associated with derivability in $\mathbf{C N L}^{\text {pol }}$, we will use the following notion of (semantic) type:

$$
\tau, \sigma::=p|\perp|(\tau \otimes \sigma) \mid \neg \tau
$$

We understand all positive atoms $p$ of $\mathbf{C N L}^{\text {pol }}$ to have been inherited, and to have been augmented by a distinguished atom $\perp$. Compound types include multiplicative products $(\tau \otimes \sigma)$ (overloading notation) and negations $\neg \tau$. Intuitively, the latter may be understood by linear negations $\tau-\circ \perp$ with $\perp$ as result.

Definition 7. Formulas $P, N$ are mapped into types $\sigma^{+}(P), \sigma^{-}(N)$, as follows: 


$$
\begin{aligned}
& \overline{\tau^{x} \vdash x: \tau} A x \\
& \frac{\Gamma \vdash s: \neg \tau \quad \Delta \vdash t: \tau}{\Gamma, \Delta \vdash(s t): \perp} \neg E \quad \frac{\Gamma, \tau^{x} \vdash s: \perp}{\Gamma \vdash \lambda x^{\tau} s: \neg \tau} \neg I \\
& \frac{\Delta \vdash t: \sigma_{1} \otimes \sigma_{2} \quad \Gamma, \sigma_{1}^{x}, \sigma_{2}^{y} \vdash s: \tau}{\Gamma, \Delta \vdash\left(\text { case } t \text { of }\left\langle x^{\sigma_{1}}, y^{\sigma_{2}}\right\rangle s\right): \tau} \otimes E \quad \frac{\Gamma \vdash s: \tau \quad \Delta \vdash t: \sigma}{\Gamma, \Delta \vdash\langle s, t\rangle: \tau \otimes \sigma} \otimes I \\
& (\lambda x s t) \rightarrow_{\beta} s[t / x] \\
& \text { case }\left\langle t_{1}, t_{2}\right\rangle \text { of }\langle x, y\rangle s \rightarrow_{\beta} s\left[t_{1} / x, t_{2} / y\right] \\
& \text { (case } \left.t \text { of }\langle x, y\rangle s s^{\prime}\right) \rightarrow_{c} \text { case } t \text { of }\langle x, y\rangle\left(s s^{\prime}\right) \\
& \text { case (case } t \text { of }\langle x, y\rangle s) \text { of }\langle u, v\rangle s^{\prime} \rightarrow_{c} \text { case } t \text { of }\langle x, y\rangle \text { (case } s \text { of }\langle u, v\rangle s^{\prime} \text { ) }
\end{aligned}
$$

Fig. 2. Target language: typing rules and reductions. The $c$-conversions correspond to the commutative conversions of Prawitz ([14]), while the 'case' notation follows that of Wadler ([15]).

$$
\begin{aligned}
\sigma^{+}(p) & =p & \sigma^{-}(\bar{p}) & =p \\
\sigma^{+}(P \otimes Q) & =\sigma^{+}(P) \otimes \sigma^{+}(Q) & \sigma^{-}(M \oplus N) & =\sigma^{-}(N) \otimes \sigma^{-}(M) \\
\sigma^{+}(\downarrow N) & =\neg \sigma^{-}(N) & \sigma^{-}(\uparrow P) & =\neg \sigma^{+}(P)
\end{aligned}
$$

If the analogy of a semantic type as capturing a possible 'kind' of denotation is to be further pursued, we require a means of describing its inhabitants.

Definition 8. F 2 defines a calculus of linear $\lambda$-terms, named $\mathbf{L} \mathbf{P}_{\otimes, \neg}$ (or simply LP) in reference to the permutative Lambek calculus. Subject of derivability are sequents $\Gamma \vdash s: \tau$, establishing inhabitation of $s$ in $\tau$ relative to a context $\Gamma$ (again overloading notation): a multiset $\left\{\tau_{1}^{x_{1}}, \ldots, \tau_{n}^{x_{n}}\right\}$ of type assignments $\tau_{1}, \ldots, \tau_{n}$ to the free variables $x_{1}, \ldots, x_{n}$ in $M$. The braces $\{$,$\} are often ommit-$ ted and we loosely write $\Gamma, \Delta$ for multiset union. Finally, sequents are to satisfy the linearity constraint that each variable in $\Gamma$ is to occur free in $s$ exactly once.

In practice, we sometimes abbreviate $\lambda z$ (case $z$ of $\langle x, y\rangle s$ ) by paired abstraction $\lambda\langle x, y\rangle s$, not to be taken as a primitive constructor.

Definition 9. The structures $\Pi, \Sigma$ of $\mathbf{C N L}^{\text {pol }}$ are built using solely positive formulas, annotated by $\mathbf{L P}$ 's variables.

$$
\Pi, \Sigma::=P^{x} \mid(\Pi \bullet \Sigma)
$$

Structures $\Pi$ map into LP contexts $\sigma(\Pi)$ by interpreting $P^{x}$ as a type assignment $\left\{\sigma^{+}(P)^{x}\right\}$ and collapsing the sole structural connective $\bullet \bullet \cdot$ into set union.

Definition 10. Figure 3 defines derivability judgements $\Pi, \Sigma \vdash s$ and $\Pi \vdash s$ : $N$ for $\mathbf{C N L}^{p o l}$, defined by mutual induction. Both carry an LP term $s$, while $N$ is said to inhabit the stoup in the latter case (adopting terminology of [5]). In practice, we will often abbreviate sequences of $\left(d p^{2}\right)$ and $\left(d p^{1}\right)$ by $(d p)$.

Sequents $\Pi, \Sigma \vdash s$ may be understood to implicitly carry $\perp$ as the type of $s$. More specifically, a straightforward induction proves 


$$
\begin{aligned}
& \frac{\Pi, \Sigma \vdash s}{p^{x} \vdash x: \bar{p}} A x \quad \frac{\Pi, \Sigma \vdash}{\Sigma, \Pi \vdash s} d p^{1} \quad \frac{\Pi \bullet \Sigma, \Upsilon \vdash s}{\overline{\Pi, \Sigma \bullet \Upsilon \vdash s}} d p^{2} \\
& \frac{\Pi \vdash s: N}{\Pi, \downarrow N^{x} \vdash(x s)} \downarrow \frac{\Pi, P^{y} \bullet Q^{z} \vdash s}{\Pi, P \otimes Q^{x} \vdash \text { case } x \text { of }\langle y, z\rangle s} \otimes \\
& \frac{\Pi, P^{x} \vdash s}{\Pi \vdash \lambda x . s: \uparrow P} \uparrow \quad \frac{\Pi \vdash s: M \quad \Sigma \vdash t: N}{\Sigma \bullet \Pi \vdash\langle t, s\rangle: M \oplus N} \oplus
\end{aligned}
$$

Fig. 3. Sequent rules for $\mathbf{C N L}^{\text {pol }}$.

Lemma 1. $\Pi, \Sigma \vdash s$ and $\Pi \vdash s: N$ imply, respectively, $\sigma(\Pi), \sigma(\Sigma) \vdash s: \perp$ and $\sigma(\Pi) \vdash s: \sigma^{-}(N)$ in $\boldsymbol{L P}$.

Lemma 2. If $\Pi, \Sigma \vdash s$ or $\Pi \vdash s: N$, then $s$ is in $\beta$-normal form.

Example 1. If formulas are categories, then structures are the binary branching trees oft encountered in linguistic analysis, while proofs are syntactic derivations. We illustrate this correspondence through the following sample expressions, involving subject relativization (1) and quantifier scope ambiguities (2).

(1) (the) lemma that confuses Hilbert

(2) A lemma confuses everyone.

To model these data, we use atomic propositions $s, n p$ and $n$, categorizing sentences, noun phrases and nouns respectively. Our lexicon assigns the following (positive) categories to the words involved:

\begin{tabular}{r|l|l} 
WorD & CATEGORY & LAMBEK CATEGORY \\
\hline Hilbert & $n p$ & $n p$ \\
everyone & $\downarrow(\uparrow n p \oplus \bar{s}) \otimes s$ & $(n p / s) \otimes s$ \\
a & $\downarrow(\uparrow n p \oplus \bar{n})$ & $n p / n$ \\
lemma & $n$ & $n$ \\
confuses & $\downarrow((\overline{n p} \oplus \uparrow s) \oplus \overline{n p})$ & $(n p \backslash s) / n p$ \\
that & $\downarrow((\bar{n} \oplus \uparrow n) \oplus \uparrow(\downarrow \bar{s} \otimes n p))$ & $(n \backslash n) /(n p \backslash s)$
\end{tabular}

For reference purposes, we included the closest corresponding traditional Lambek formulas, with forward and backward implications $A / B$ and $B \backslash A$ understood as $A \oplus B^{\perp}$ and $B^{\perp} \oplus A$ respectively $\left.\right|^{2}$ While the category for 'everyone' may appear unconventional, the key idea is to have it include a subformula of the form $\downarrow N$, allowing $(\downarrow)$ to fix its scope. Its contractibility to $n p$ is what further sets it apart from the commonly used traditional category $s /(n p \backslash s)$. Figures 5 and 6 provide derivations for (1) and (2), using $\mathrm{F} 4$ s derivation of transitive clauses as a macro.

The terms derived in Figures 5 and 6 constitute the denotations of the expressions (1) and (2), parameterized over the denotations of the words contained therein via the use of free variables. Note in particular the derivation of distinct terms for (2), corresponding to the two available scopal readings.

\footnotetext{
${ }^{2}$ [7] demonstrates CNL embeds (intuitionistic) non-associative Lambek calculus.
} 


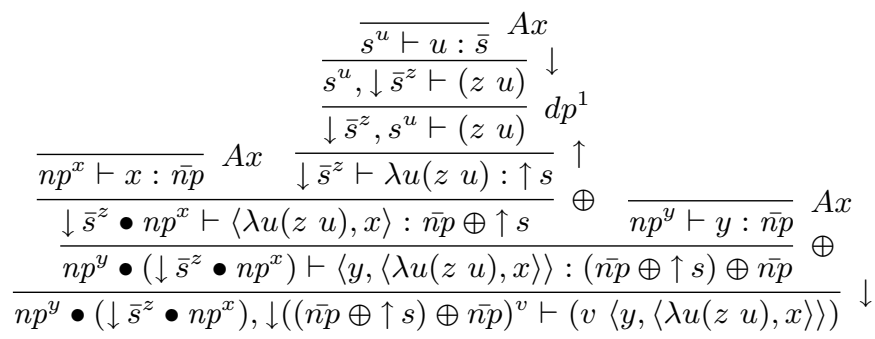

Fig. 4. A 'macro' for deriving transitive clauses.

\subsection{Lexical Semantics}

The previous section left us with the task of instantiating the lexical parameters feeding the denotations found in Figures 5 and 6 . In doing so, we will admit ourselves some leverage in the strictness of the resource management regime adopted thus far: while the 'derivational' semantics of $\S 3.2$ was meant to reflect the linearity of the source $\mathbf{C N L}^{\text {pol }}$, the lexical semantics, constituting our means of referring to the world around us, need not be so restricted.

Put in more precise terms, in filling the lexical gap left by $\S 3.2$, we permit access to the full simply-typed $\lambda$-calculus, augmented by the logical constants of first-order predicate logic. To start with, the type language is revised thus:

$$
\tau, \sigma::=e|t|(\sigma \rightarrow \tau) \mid(\sigma \times \tau)
$$

We have adopted base types $e$ and $t$, interpreting, respectively, a fixed set of 'entities', or discourse referents, and the Boolean truth values. Complex types are either implications or products, the latter allowing the formation of pairs $\langle s, t\rangle$ and the left- and right projections $\pi_{1}(s)$ and $\pi_{2}(s)$. In updating our lexical entries, we associate words of category $P$ with terms of type $\sigma^{+}(P)$, 'delinearized' by systematically replacing $\perp,(\tau \otimes \sigma)$ and $\neg \tau$ with $t, \tau \times \sigma$ and $\tau \rightarrow t$ respectively:

\begin{tabular}{r|l|l} 
Word & CATEGORY & DenOtations \\
\hline Hilbert & $n p$ & hilbert \\
everyone & $\downarrow(\uparrow n p \oplus \bar{s}) \otimes s$ & $\langle\lambda\langle p, P\rangle \forall y(($ person $y) \Rightarrow(P y)) \wedge p, \top\rangle$ \\
$\mathrm{a}$ & $\downarrow(\uparrow n p \oplus \bar{n})$ & $\lambda\langle Q, P\rangle \exists x((Q x) \wedge(P x))$ \\
lemma & $n$ & lemma \\
confuses & $\downarrow((\overline{n p} \oplus \uparrow s) \oplus \overline{n p})$ & $\lambda\langle x,\langle q, y\rangle\rangle(q(($ confuse $y) x))$ \\
that & $\downarrow((\bar{n} \oplus \uparrow n) \oplus \uparrow(\downarrow \bar{s} \otimes n p))$ & $\lambda\langle R,\langle Z, Q\rangle\rangle(Z \lambda z((Q z) \wedge(R\langle\lambda p p, z\rangle)))$
\end{tabular}

Here, $\lambda\langle y, z\rangle t$ abbreviates $\lambda x^{\tau \times \sigma} t\left[\pi_{1}(x) / y, \pi_{2}(x) / z\right]$. Note the use of nonlogical constants hilbert, person, lemma and confuse, of types $e, e \rightarrow t(\times 2)$ and $e \rightarrow(e \rightarrow t)$ respectively. Like types, the linear terms of $\$ 3.2$ carry over straightforwardly: just replace case analyses case $s$ of $\langle x, y\rangle t$ with projections $t\left[\pi_{1}(s) / x, \pi_{2}(s) / y\right]$. Substitution of the above lexical denotations for the appropriate variables then yields the following terms: 


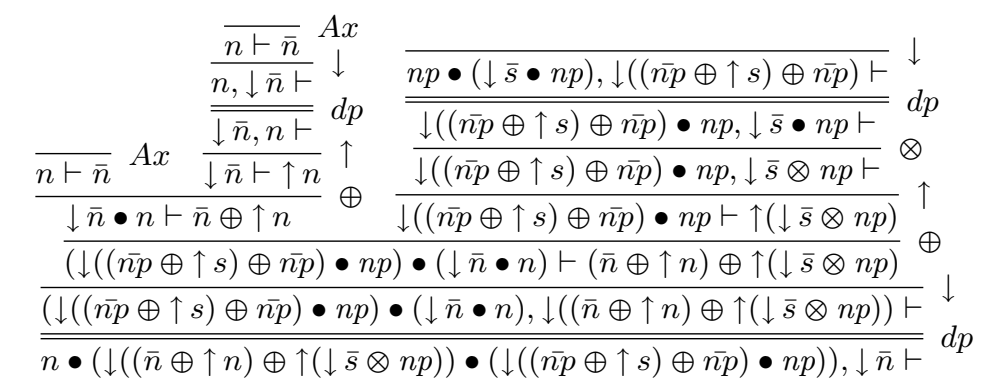

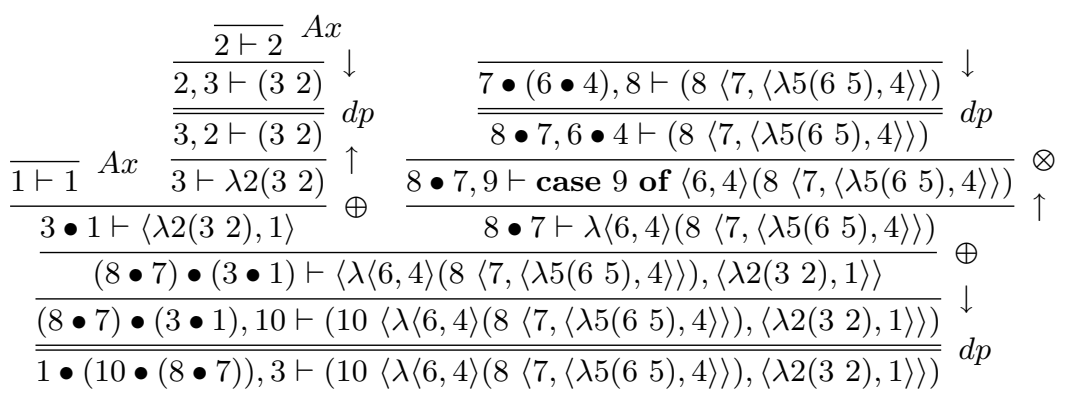

Fig. 5. Derivation illustrating subject relativization. Reasons of space dictate the spreading of the syntactic and semantic components over separate derivation trees. Furthermore, due to the number of variables involved in the semantics, we use numbers as names (not to be confused with De Bruijn notation).
(1) $(3 \lambda z(($ lemma $z) \wedge(($ confuse hilbert $) z)))$
(2a) $\forall y(($ person $y) \Rightarrow \exists x(($ lemma $x) \wedge(3(($ confuses $y) x)))) \wedge \top)$
(2b) $\exists x(($ lemma $x) \wedge(\forall y(($ person $y) \Rightarrow(3(($ confuses $y) x))) \wedge \top))$

In each case, we are left with a free variable 3, corresponding to the category of the whole expression. Also, for $(2 \mathrm{a})$ and $(2 \mathrm{~b})$, note $\phi \wedge \top$ is logically equivalent with $\phi$. Alternatively, one may use the more complex category $\downarrow(\uparrow n p \oplus \uparrow s) \otimes \downarrow \bar{s}$ for 'everyone', with denotation $\lambda\langle q, P\rangle \forall y(q(($ person $y) \Rightarrow(P y))), \lambda p p\rangle$.

\section{Comparing provability in $\mathrm{CNL}^{\text {pol }}$ and CNL}

We defined polarized CNL and briefly demonstrated its application to formal semantics. Suffice it to assure that $\mathbf{C N L}$ and $\mathbf{C N L}^{\text {pol }}$ coincide on provability. One direction is straightforward: a derivation in $\mathbf{C N L}^{p o l}$ is easily rewritten into one of CNL by dropping the shifts. Conversely, we witness a decoration of CNL formulae with shifts $\uparrow, \downarrow$, generating no (sub)formulas of the form $\downarrow \uparrow P$ or $\uparrow \downarrow N$, s.t. provability in CNL implies provability in $\mathbf{C N L}^{\text {pol }}$ w.r.t. said decoration.

The bulk of this section is devoted to proving closure of $\mathbf{C N L}^{\text {pol }}$ under Cut, using an argument involving phase spaces ([13]). Said models are defined in $\S 4.1$, together with proofs of soundness and completeness. $\S 4.2$ uses the results of $\S 4.1$ 

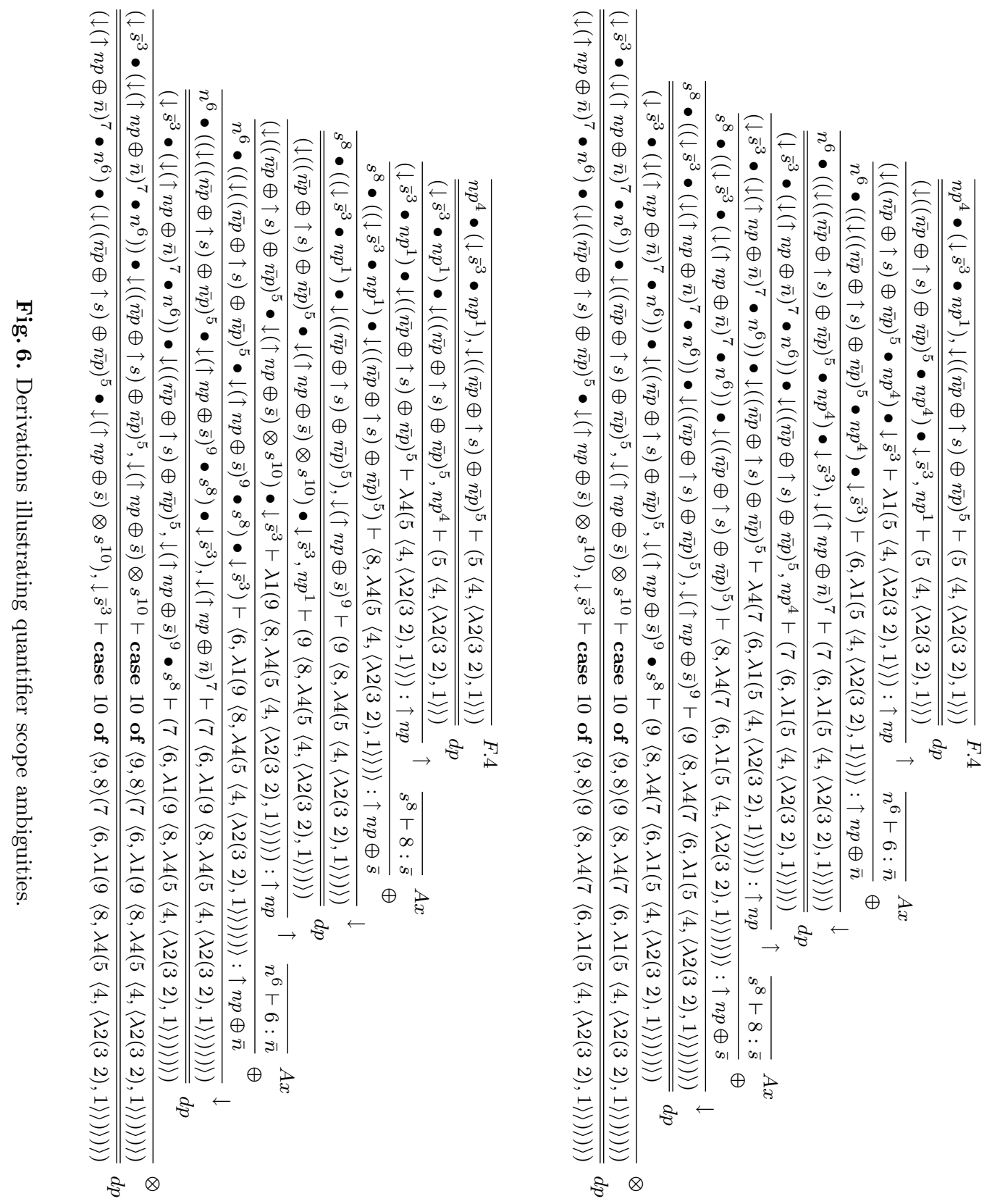
to prove Cut admissibility, and provides the decoration by which we demonstrate the completeness of $\mathbf{C N L}^{\text {pol }}$ w.r.t. CNL. In what is to follow, we will only be concerned with provability, hence omit any mention of term annotations.

\subsection{Phase spaces}

Definition 11. A phase space is a 3-tuple $\langle P, \bullet, \perp\rangle$ where:

1. $P$ is a non-empty set of phases closed under $\bullet: P \times P \rightarrow P$.

2. $\perp \subseteq P \times P$ s.t. $\forall x, y, z \in P$,

$$
\begin{gathered}
\langle x, y\rangle \in \perp \Rightarrow\langle y, x\rangle \in \perp \\
\langle x \bullet y, z\rangle \in \perp \Leftrightarrow\langle x, y \bullet z\rangle \in \perp
\end{gathered}
$$

As usual, we often identify a phase space by its carrier set $P$. Define $\cdot^{\perp}: \mathscr{P}(P) \rightarrow$ $\mathscr{P}(P)$ by $A \mapsto\{x \mid(\forall y \in A)(\langle x, y\rangle \in \perp)\}$.

Lemma 3. Given $P$, one easily shows $A \subseteq B^{\perp}$ iff $B \subseteq A^{\perp}(A, B \in \mathscr{P}(P))$. In other words, $\cdot^{\perp}$ is a Galois connection, and hence $\cdot^{\perp \perp}$ a closure operator.

Formulas will be interpreted by facts: subsets $A \subseteq P$ s.t. $A=A^{\perp \perp}$.

Definition 12. A model consists of a phase space $P$ and a valuation $v$ taking positive atoms $p$ into facts. $v$ extends to maps $v^{+}(\cdot)$ and $v^{-}(\cdot)$, defined by mutual induction and acting on arbitrary positive and negative formulas respectively:

$$
\begin{aligned}
v^{+}(p) & :=v(p) & v^{-}(\bar{p}) & :=v(p) \\
v^{+}(P \otimes Q) & :=v^{+}(P) \times v^{+}(Q) & v^{-}(M \oplus N) & :=v^{-}(N) \times v^{-}(M) \\
v^{+}(\downarrow N) & :=v^{-}(N)^{\perp} & v^{-}(\uparrow P) & :=v^{+}(P)^{\perp}
\end{aligned}
$$

Here, $\times: \mathscr{P}(P) \times \mathscr{P}(P) \rightarrow \mathscr{P}(P)$ is defined $A \times B:=\left\{x \bullet y \mid x \in A^{\perp}, y \in B^{\perp}\right\}^{\perp}$. Since Lemma 3 implies $A \subseteq A^{\perp \perp}$ and $A^{\perp \perp \perp} \subseteq A^{\perp}$ for $A \subseteq P$, this set is a fact.

Lemma 4. $v^{+}(P)=v^{-}(\uparrow P)^{\perp}$ and $v^{-}(N)=v^{+}(\downarrow N)^{\perp}$ for any $N, P$.

Proof. Immediate, since the sets involved are facts.

Lemma 5. For any $N, P, v^{+}(P)=v^{-}\left(P^{\perp}\right)$ and (dually) $v^{-}(N)=v^{+}\left(N^{\perp}\right)$.

Proof. By a straightforward inductive argument.

Definition 13. To state soundness and completeness, we interpret a structure $\Pi$ by dual formulas $\Pi^{+}$and $\Pi^{-}$, in the sense that $\Pi^{+\perp}=\Pi^{-}$and $\Pi^{-\perp}=\Pi^{+}$:

$$
\begin{aligned}
P^{+} & =P & P^{-} & =P^{\perp} \\
(\Pi \bullet \Sigma)^{+} & =\Pi^{+} \otimes \Sigma^{+} & (\Pi \bullet \Sigma)^{-} & =\Sigma^{-} \oplus \Pi^{-}
\end{aligned}
$$

Lemma 6. We have the following equivalences:

$$
\begin{aligned}
& v^{-}\left(\uparrow \Pi^{+}\right) \subseteq v^{-}\left(\Sigma^{-}\right) \Leftrightarrow v^{-}\left(\uparrow \Sigma^{+}\right) \subseteq v^{-}\left(\Pi^{-}\right) \\
& \Leftrightarrow v^{+}\left(\downarrow \Sigma^{-}\right) \subseteq v^{+}\left(\Pi^{+}\right) \Leftrightarrow v^{+}\left(\downarrow \Pi^{-}\right) \subseteq v^{+}\left(\Sigma^{+}\right)
\end{aligned}
$$


Proof. Recalling $\Upsilon^{+\perp}=\Upsilon^{-}$and $\Upsilon^{-\perp}=\Upsilon^{+}$for arbitrary $\Upsilon$, we have

$$
\begin{array}{llll}
v^{+}\left(\downarrow \Sigma^{-}\right) \subseteq v^{+}\left(\Pi^{+}\right) & \text {iff } & v^{+}\left(\Pi^{+}\right)^{\perp} \subseteq v^{+}\left(\downarrow \Sigma^{-}\right)^{\perp} & \text { (Lemma 3) } \\
& \text { iff } & v^{-}\left(\uparrow \Pi^{+}\right) \subseteq v^{-}\left(\Sigma^{-}\right) & \text {(Lemma }
\end{array}
$$

and

$$
\begin{array}{rlll}
v^{+}\left(\downarrow \Sigma^{-}\right) \subseteq v^{+}\left(\Pi^{+}\right) & \text {iff } & v^{+}\left(\Pi^{+}\right)^{\perp} \subseteq v^{+}\left(\downarrow \Sigma^{-}\right)^{\perp} & \text { (Lemma 3) } \\
& \text { iff } & v^{-}\left(\Pi^{-}\right)^{\perp} \subseteq v^{-}\left(\uparrow \Sigma^{+}\right)^{\perp} & \text { (Lemma } 5 \\
& \text { iff } & v^{+}\left(\downarrow \Pi^{-}\right) \subseteq v^{+}\left(\Sigma^{+}\right) & \text {(Lemma 4 }
\end{array}
$$

and similarly $v^{-}\left(\uparrow \Pi^{+}\right) \subseteq v^{-}\left(\Sigma^{-}\right)$iff $v^{-}\left(\uparrow \Sigma^{+}\right) \subseteq v^{-}\left(\Pi^{-}\right)$.

Theorem 14. All phase models satisfy the following implications:

$$
\begin{aligned}
& \Pi, \Sigma \vdash \Longrightarrow v^{+}\left(\downarrow \Pi^{-}\right) \subseteq v^{+}\left(\Sigma^{+}\right) \\
& \Pi \vdash N \Longrightarrow v^{-}(N) \subseteq v^{-}\left(\Pi^{-}\right)
\end{aligned}
$$

Proof. By induction on the derivation witnessing $\Pi, \Sigma \vdash$ or $\Pi \vdash N$, freely making use of L, 6 . The only nontrivial cases are $(\oplus)$ and $\left(d p^{2}\right)$.

Case $(\oplus)$. Since $v^{-}\left(\Pi^{-}\right)^{\perp} \subseteq v^{-}(M)^{\perp}$ and $v^{-}\left(\Sigma^{-}\right)^{\perp} \subseteq v^{-}(N)^{\perp}$ by the induction hypothesis and L 3 , we have

$$
\left\{y \bullet x \mid x \in v^{-}\left(\Pi^{-}\right)^{\perp}, y \in v^{-}\left(\Sigma^{-}\right)^{\perp}\right\} \subseteq\left\{y \bullet x \mid x \in v^{-}(M)^{\perp}, y \in v^{-}(N)^{\perp}\right\}
$$

with another application of L 3 ensuring $v^{-}(M \oplus N) \subseteq v^{-}\left(\Pi^{-} \oplus \Sigma^{-}\right)$.

Case $\left(d p^{2}\right)$. We check one direction, establishing

$$
\frac{\left(\left(v^{+}\left(\Sigma^{+}\right) \times v^{+}\left(\Upsilon^{+}\right)\right)^{\perp}=\right) v^{-}\left(\uparrow(\Sigma \bullet \Upsilon)^{+}\right) \subseteq v^{-}\left(\Pi^{-}\right)}{v^{-}\left(\uparrow \Upsilon^{+}\right) \subseteq v^{-}\left((\Pi \bullet \Sigma)^{-}\right)\left(=\left\{x \bullet y \mid x \in v^{-}\left(\Pi^{-}\right)^{\perp}, y \in v^{-}\left(\Sigma^{-}\right)^{\perp}\right\}^{\perp}\right)}
$$

applying $\mathrm{L} 6$ twice to obtain the desired result. Thus, it suffices to prove $\langle z, x \bullet$ $y\rangle \in \perp$ on the assumptions (a) $z \in v^{-}\left(\uparrow \Upsilon^{+}\right)$; (b) $x \in v^{-}\left(\Pi^{-}\right)^{\perp}$; and (c) $y \in v^{-}\left(\Sigma^{-}\right)^{\perp}$. By applying $\mathrm{L} 3$ on the induction hypothesis, (b) implies $x \in$ $v^{+}\left(\Sigma^{+}\right) \times v^{+}\left(\Upsilon^{+}\right)=\left\{y \bullet z \mid y \in v^{+}\left(\Sigma^{+}\right)^{\perp}, z \in v^{+}\left(\Upsilon^{+}\right)^{\perp}\right\}^{\perp}$. By (a), (c) and Lemmas 4 and $5, y \in v^{+}\left(\Sigma^{+}\right)^{\perp}$ and $z \in v^{+}\left(\Upsilon^{+}\right)^{\perp}$, so that $\langle x, y \bullet z\rangle \in \perp$, iff $\langle z, x \bullet y\rangle \in \perp$.

Definition 15. Completeness will be established w.r.t. the syntactic (phase) model, defined by taking the structures $\Pi$ as phases, setting $\langle\Pi, \Sigma\rangle \in \perp$ iff $\Pi, \Sigma \vdash$ and letting $v(p)=\{p\}^{\perp}=\{\Pi \mid \Pi, p \vdash\}$.

The following is our central lemma, resembling results of Okada (13]) and Herbelin and Lee ([10]) for linear and intuitionistic logic respectively.

Lemma 7. For arbitrary $P, N, \Pi, \Sigma$, the syntactic model satisfies:

(i) $\Pi \in v^{-}(N)$ implies $\Pi, N^{\perp} \vdash$

(ii) $(\forall \Pi)(\Pi \vdash N \Rightarrow \Pi, \Sigma \vdash)$

implies $\Sigma \in v^{-}(N)$ (iii) $\Pi \in v^{+}(P)$ implies $\Pi, P \vdash$

(iv) $(\forall \Pi)\left(\Pi \vdash P^{\perp} \Rightarrow \Pi, \Sigma \vdash\right)$

implies $\Sigma \in v^{+}(P)$ 
Proof. First, note that if $\Pi \vdash N\left(\Pi \vdash P^{\perp}\right)$, then also $\Pi, \downarrow N \vdash\left(\Pi, \downarrow P^{\perp} \vdash\right)$ by applying ( $\downarrow$ ). Consequently, (ii) and (iv) imply, respectively, $\downarrow N \in v^{-}(N)$ and $\downarrow P^{\perp} \in v^{+}(P)$. In practice, when invoking the induction hypothesis for (ii) or (iv), we often immediately instantiate them by the latter consequences. To prove (i)-(iv), we proceed by a simultaneous induction on $P, N$. We suffice by checking the cases $\bar{p}, \uparrow P$ and $M \oplus N$, the remaining $p, \downarrow N$ and $P \otimes Q$ being entirely dual.

Case $\bar{p}$. We show (i) and (ii).

(i) Since $v^{-}(\bar{p})=\{p\}^{\perp}, \Pi \in v^{-}(p)$ implies $\Pi, p \vdash$ by definition.

(ii) $\Pi \vdash \bar{p}$ iff $\Pi=p$, and $p, \Sigma \vdash$ again implies $\Sigma \in v^{-}(\bar{p})$ by definition.

Case $\uparrow P$. We show (i) and (ii).

(i) Suppose $\Pi \in v^{-}(\uparrow P)=v^{+}(P)^{\perp}$. By IH(iv), $\downarrow P^{\perp} \in v^{+}(P)$, so that $\left\langle\Pi, \downarrow P^{\perp}\right\rangle \in$ $\perp$, and hence $\Pi, \downarrow P^{\perp} \vdash$ by definition of $\perp$.

(ii) We show $\Sigma \in v^{-}(\uparrow P)=v^{+}(P)^{\perp}$, assuming (a) $\Pi \vdash \uparrow P$ implies $\Pi, \Sigma \vdash$ for all $\Pi$. Letting (b) $\Upsilon \in v^{+}(P)$, it suffices to ensure $\Sigma, \Upsilon \vdash$. IH(iii) and (b) imply $\Upsilon, P \vdash$, hence $\Upsilon \vdash \uparrow P$ by ( $\uparrow)$. Thus, $\Upsilon, \Sigma \vdash$ by (a), and we apply $\left(d p^{1}\right)$.

Case $M \oplus N$. We show (i) and (ii).

(i) Let (a) $\Pi \in v^{-}(M \oplus N)$. We show $\Pi, N^{\perp} \bullet M^{\perp} \vdash$, implying $\Pi, N^{\perp} \otimes M^{\perp} \vdash$ by $(\otimes)$. By (a), it suffices to ensure $M^{\perp} \in v^{-}(M)^{\perp}$ and $N^{\perp} \in v^{-}(N)^{\perp}$. I.e., we must ascertain $\Sigma, M^{\perp} \vdash$ and $\Upsilon, N^{\perp} \vdash$ on the assumptions $\Sigma \in v^{-}(M)$ and $\Upsilon \in v^{-}(N)$, but these are immediate consequences of $\mathrm{IH}(\mathrm{i})$ and $\left(d p^{2}\right)$.

(ii) The following hypotheses will be used:

(a) $\Pi \vdash M \oplus N$ implies $\Pi, \Sigma \vdash$ for all $\Pi$

(b) $\Upsilon_{1} \in v^{-}(M)^{\perp}$

(c) $\Upsilon_{2} \in v^{-}(N)^{\perp}$

(d) $\left(\forall \Pi_{1}\right)\left(\Pi_{1} \vdash M \Rightarrow \Pi_{1}, \Sigma \bullet \Upsilon_{2} \vdash\right)$ implies $\Sigma \bullet \Upsilon_{2} \in v^{-}(M)$

(e) $\Pi_{1} \vdash M$

(f) $\left(\forall \Pi_{2}\right)\left(\Pi_{2} \vdash N \Rightarrow \Pi_{2}, \Pi_{1} \bullet \Sigma \vdash\right)$ implies $\Pi_{1} \bullet \Sigma \in v^{-}(M)$

(g) $\Pi_{2} \vdash N$

Assuming (a), we show $\Sigma \in v^{-}(M \oplus N)$, iff $\Sigma, \Upsilon \vdash$ for all $\Upsilon \in\left\{\Upsilon_{2} \bullet \Upsilon_{1} \mid \Upsilon_{1} \in\right.$ $\left.v^{-}(M)^{\perp}, \Upsilon_{2} \in v^{-}(N)^{\perp}\right\}$. So assume (b), (c). Since $\Sigma, \Upsilon_{2} \bullet \Upsilon_{1} \vdash$ iff $\Sigma \bullet \Upsilon_{2}, \Upsilon_{1} \vdash$ by $\left(d p^{2}\right)$, it suffices by (b) to prove $\Sigma \bullet \Upsilon_{2} \in v^{-}(M)$. By (d), i.e., IH(ii), we need only prove $\Pi_{1}, \Sigma \bullet \Upsilon_{2} \vdash$ on the assumption (e), iff $\Pi_{1} \bullet \Sigma, \Upsilon_{2} \vdash$ by $\left(d p^{2}\right)$. Applying (c), we must show $\Pi_{1} \bullet \Sigma \in v^{-}(M)$, which follows from (f), i.e., IH(ii), if we can prove $\Pi_{2}, \Pi_{1} \bullet \Sigma \vdash$ on the assumption (g). By $\left(d p^{2}\right)$ and (a), this follows from $\Pi_{2} \bullet \Pi_{1} \vdash M \oplus N$, witnessed by (e), (g) and ( $\oplus$ ).

Lemma 8. We have the following implications:

(i) $v^{+}\left(\Sigma^{+}\right) \subseteq\{\Pi \mid \Pi, \Sigma \vdash\}$ implies $\Sigma \in v^{-}\left(\uparrow \Sigma^{+}\right)$

(ii) $v^{-}\left(\Sigma^{-}\right) \subseteq\{\Pi \mid \Pi, \Sigma \vdash\}$ implies $\Sigma \in v^{+}\left(\downarrow \Sigma^{-}\right)$ 
Proof. We show (i), with (ii) being dual:

$$
\begin{array}{rlrl} 
& v^{+}\left(\Sigma^{+}\right) \subseteq\{\Pi \mid \Pi, \Sigma \vdash\} \\
\Leftrightarrow & \{\Pi \mid \Pi, \Sigma \vdash\}^{\perp} \subseteq v^{+}\left(\Sigma^{+}\right)^{\perp} & \text { (L) 3 } \\
\Leftrightarrow & \{\Upsilon \mid(\forall \Pi)(\Pi, \Sigma \vdash \Rightarrow \Upsilon, \Pi \vdash)\} \subseteq v^{-}\left(\uparrow \Sigma^{+}\right) & \text {(def.) }
\end{array}
$$

And evidently $\Sigma \in\{\Upsilon \mid(\forall \Pi)(\Pi, \Sigma \vdash \Rightarrow \Upsilon, \Pi \vdash)\}$.

Lemma 9. We have (i) $\Pi \in v^{+}\left(\Sigma^{+}\right)$implies $\Pi, \Sigma \vdash$; and (ii) $\Pi \in v^{-}\left(\Sigma^{-}\right)$ implies $\Pi, \Sigma \vdash$ for arbitrary $\Sigma, \Pi$.

Proof. By induction on $\Sigma$. The base case reduces to (i) and (iii) of L7, For $\Sigma=\Sigma_{1} \bullet \Sigma_{2}$, we prove (i), with (ii) being similar. So let $\Pi \in v^{+}\left(\Sigma_{1}^{+} \otimes \Sigma_{2}^{+}\right)$. The desired result follows if we can show $\Sigma_{1} \in v^{+}\left(\Sigma_{1}^{+}\right)^{\perp}=v^{-}\left(\uparrow \Sigma_{1}^{+}\right)$and $\Sigma_{2} \in v^{+}\left(\Sigma_{2}^{+}\right)^{\perp}=v^{-}\left(\uparrow \Sigma_{2}^{+}\right)$. But this holds by virtue of IH(i) and L $8(\mathrm{i})$.

Theorem 16. If $v^{+}\left(\downarrow \Pi^{-}\right) \subseteq v^{+}\left(\Sigma^{+}\right)$in the syntactic model, then $\Pi, \Sigma \vdash$.

Proof. By L $8 \& 9, \Pi \in v^{+}\left(\downarrow \Pi^{-}\right)$, hence $\Pi \in v^{+}\left(\Sigma^{+}\right)$, so $\Pi, \Sigma \vdash$ by L 9

\subsection{Cut admissibility and completeness w.r.t. CNL}

We proceed with the completeness proof for derivability in $\mathbf{C N L}^{\text {pol }}$ w.r.t. CNL, to be witnessed by the following decoration of CNL formulae with shifts.

Definition 17. For $A$ a formula of CNL, let $\epsilon(A)=+$ if $A$ is of the form $p$ or $B \otimes C$, and $\epsilon(A)=-$ otherwise. We translate $A$ into a formula $\uparrow(A)$ of $\mathbf{C N L}^{\text {pol }}$ with no subformulas of the form $\downarrow \uparrow P$ or $\uparrow \downarrow N$. In the base cases, $\uparrow(p)=p$, $\uparrow(\bar{p})=\bar{p}$, while for complex formulae,

\begin{tabular}{|cc|l|l|}
\hline$\epsilon(A)$ & $\epsilon(B)$ & $\uparrow(A \otimes B)$ & $\uparrow(A \oplus B)$ \\
\hline+ & + & $\uparrow(A) \otimes \uparrow(B)$ & $\uparrow \uparrow(A) \oplus \uparrow \uparrow(B)$ \\
+ & - & $\uparrow(A) \otimes \downarrow \uparrow(B)$ & $\uparrow \uparrow(A) \oplus \uparrow(B)$ \\
- & + & $\downarrow \uparrow(A) \otimes \uparrow(B)$ & $\uparrow(A) \oplus \uparrow \uparrow(B)$ \\
- & - & $\downarrow \uparrow(A) \otimes \downarrow \uparrow(B)$ & $\uparrow(A) \oplus \uparrow(B)$ \\
\hline
\end{tabular}

Definition 18. The map $\uparrow(\cdot)$ is extended to the level of structures $\Gamma$ as follows:

$$
A \mapsto\left\{\begin{array}{rl}
\uparrow(A) & \text { if } \epsilon(A)=+ \\
\downarrow \uparrow(A) & \text { if } \epsilon(A)=-
\end{array} \quad \uparrow(\Gamma \bullet \Delta)=\uparrow(\Gamma) \bullet \uparrow(\Delta)\right.
$$

Our intention is to show that $\Gamma, \Delta \vdash$ implies $\uparrow(\Gamma), \uparrow(\Delta) \vdash$.

Lemma 10. The following rules are admissible for polarized $\boldsymbol{C N L}$ :

$$
\overline{\downarrow N, N^{\perp} \vdash} \quad \frac{\Sigma, P \vdash \quad \Pi, \downarrow P^{\perp} \vdash}{\Pi, \Sigma \vdash}
$$

Proof. L 7 (ii) implies $\downarrow N \in v^{-}(N)$, so that $\downarrow N, N^{\perp} \vdash$ by L 7 (i). Now suppose $\Pi, \downarrow P^{\perp} \vdash$ and $\Sigma, P \vdash$. By T $14, v^{+}\left(\downarrow \Pi^{-}\right) \subseteq v^{+}\left(\downarrow P^{\perp}\right)$ and $v^{+}\left(\downarrow P^{\perp}\right) \subseteq v^{+}\left(\Sigma^{+}\right)$, hence $v^{+}\left(\downarrow \Pi^{-}\right) \subseteq v^{+}\left(\Sigma^{+}\right)$so that $\Pi, \Sigma \vdash$ by T 16 . 
Lemma 11. Let $(\cdot)^{\bullet}$ take positive formulas into structures, as follows:

$$
(p)^{\bullet}=p ; \quad(\downarrow N)^{\bullet}=\downarrow N ; \quad(P \otimes Q)^{\bullet}=(P)^{\bullet} \bullet(Q)^{\bullet}
$$

then (i) $\Pi,(P)^{\bullet} \vdash$ implies $\Pi, P \vdash$; and (ii) $\left(N^{\perp}\right)^{\bullet} \vdash N$, for any $P, N$.

Proof. We prove (i) by induction on $P$. If $P=p$ or $P=\downarrow N$, the desired result is immediate. If $P=P_{1} \otimes P_{2}$, proceed as follows:

$$
\begin{aligned}
& \frac{\Pi,\left(P_{1}\right)^{\bullet} \bullet\left(P_{2}\right) \vdash}{\left(P_{2}\right)^{\bullet} \bullet \Pi,\left(P_{1}\right) \vdash} d p^{1}, d p^{2}, d p^{1} \\
& \frac{\Pi \bullet P_{1},\left(P_{2}\right)^{\bullet} \vdash}{\Pi, P_{1} \bullet P_{2} \vdash} I H, d p^{2}, d p^{1} \\
& \frac{\Pi, d p^{2}}{\Pi, P_{1} \otimes P_{2} \vdash} \otimes
\end{aligned}
$$

Similarly, we prove (ii) by induction on $N$ :

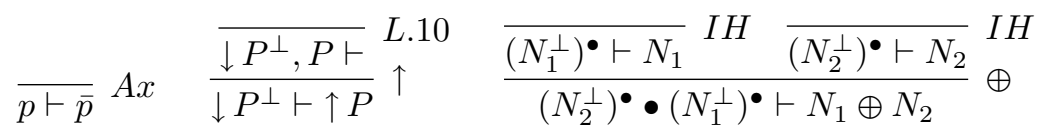

$$
\begin{aligned}
& (N=\bar{p}) \quad(N=\uparrow P) \quad\left(N=N_{1} \oplus N_{2}\right)
\end{aligned}
$$

Theorem 19. $\Gamma, \Delta \vdash$ in $\boldsymbol{C N L}$ implies $\uparrow(\Gamma), \uparrow(\Delta) \vdash$ in $\boldsymbol{C N L}^{\text {pol }}$.

Proof. We proceed by induction on the derivation establishing $\Gamma, \Delta \vdash$. The cases $(A x)$ and $(C u t)$ follow immediately from $\mathrm{C} 10$, possibly with some applications of $\left(d p^{1}\right)$ depending on the value of $\epsilon(A)$. The case $(\otimes)$ is equally trivial, translating to an application of $(\otimes)$ in $\mathbf{C N L}^{\text {pol }}$. Thus, we are left to check

Case $(\oplus)$. Suppose $\Gamma, A \vdash$ and $\Delta, B \vdash$. Considering all possible values for $\epsilon(A)$ and $\epsilon(B)$, we have four subcases to check in total. As a typical case, we pick $\epsilon(A)=-$ and $\epsilon(B)=+$. Thus, by induction hypothesis, $\uparrow(\Gamma), \downarrow \uparrow(A) \vdash$ and $\uparrow(\Delta), \uparrow(B) \vdash$. We construct a derivation of $\uparrow(\Delta) \bullet \uparrow(\Gamma), \downarrow(A \oplus \uparrow B) \vdash$ as follows:

$$
\begin{aligned}
& \overline{\left(\uparrow(A)^{\perp}\right)^{\bullet} \vdash A} L \underline{11} \text { (ii) } \frac{\overline{\downarrow \uparrow(B)^{\perp}, B \vdash}}{\frac{\downarrow \uparrow(B)^{\perp} \vdash \downarrow B}{10}} \uparrow \\
& \frac{\downarrow \uparrow(B)^{\perp} \bullet\left(\uparrow(A)^{\perp}\right)^{\bullet} \vdash A \oplus \uparrow B}{\downarrow \uparrow(B)^{\perp} \bullet\left(\uparrow(A)^{\perp}\right)^{\bullet}, \downarrow(A \oplus \uparrow B) \vdash} \downarrow \\
& \frac{\downarrow \downarrow \downarrow}{\downarrow(A \oplus \uparrow B) \bullet \downarrow \uparrow(B)^{\perp},\left(\uparrow(A)^{\perp}\right)^{\bullet} \vdash} d p^{1}, d p^{2}
\end{aligned}
$$

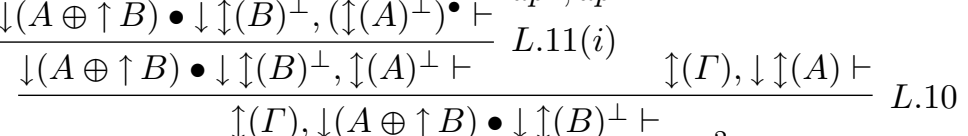

$$
\begin{aligned}
& \frac{\uparrow(\Delta), \uparrow(B) \vdash \quad \frac{}{\uparrow(\Gamma) \bullet \downarrow(A \oplus \uparrow B), \downarrow \uparrow(B)^{\perp} \vdash}}{\uparrow} L \underline{10} \\
& \frac{\uparrow(\Gamma) \bullet \downarrow(A \oplus \uparrow B), \uparrow(\Delta) \vdash}{\uparrow(\Delta) \bullet \uparrow(\Gamma), \downarrow(A \oplus \uparrow B) \vdash} d p^{1}, d p^{2}
\end{aligned}
$$




\section{Related Topics}

We consider some related topics and directions for future research.

\subsection{Focused proof search}

Though not made explicit in their choice of terminology, Hepple ([9]) and Hendriks ([8], Ch.4) were the first to study focused proof search within the Lambek calculus, with the aim of eliminating spurious ambiguities from Cut-free derivations. Strictly speaking, $\mathbf{C N L}^{\mathrm{pol}}$ 's derivations do not fully conform to Andreoli's specifications, as the latter enforces the eager application of invertible inferences. In the lower derivation of $\mathrm{F} 6$, however, nothing prevents us from postponing the application of $(\otimes)$ until right before the second application of $(\oplus)$ (adopting a top-down view). For the moment we have ignored such refinements, seeing as they have no bearing on the matter of finding a formal semantics.

\subsection{The Lambek-Grishin calculus}

The ideas expressed in this article are not limited to CNL. To illustrate, we briefly discuss a proposal similar to that of De Groote and Lamarche, namely the Lambek-Grishin calculus of Moortgat and associates (LG, 12]). We sketch a polarized reinterpretation of $\mathbf{L G}$ (henceforth $\mathbf{L} \mathbf{G}^{\text {pol }}$ ), adopting a syntax highlighting the similarities with CNL, though rather different from Moortgat's. Compared to CNL, LG's formulae include explicit connectives for implications $/, \backslash$ and (the dual) subtractions $\varnothing, \oslash$.

$$
\begin{gathered}
P, Q::=p|(P \otimes Q)|(P \oslash M)|(M \otimes P)|(\downarrow N) \\
M, N::=\bar{p}|(M \oplus N)|(P \backslash M)|(M / P)|(\uparrow P)
\end{gathered}
$$

Moortgat's original (two-sided) account of LG lacks negative atoms, added here for the purpose of allowing classical negation to be defined:

$$
\begin{aligned}
& p^{\perp}=\bar{p} \quad \bar{p}^{\perp}=p \\
& (P \otimes Q)^{\perp}=Q^{\perp} \oplus P^{\perp} \quad(M \oplus N)^{\perp}=N^{\perp} \otimes M^{\perp} \\
& (P \oslash M)^{\perp}=M^{\perp} \backslash P^{\perp} \quad(P \backslash M)^{\perp}=M^{\perp} \oslash P^{\perp} \\
& (M \otimes P)^{\perp}=P^{\perp} / M^{\perp} \quad(M / P)^{\perp}=P^{\perp} \otimes M^{\perp} \\
& (\downarrow N)^{\perp}=\uparrow N^{\perp} \quad(\uparrow P)^{\perp}=\downarrow P^{\perp}
\end{aligned}
$$

The category-type correspondence is adapted straightforwardly:

$$
\begin{aligned}
\sigma^{+}(p) & =p & \sigma^{-}(\bar{p}) & =p \\
\sigma^{+}(P \otimes Q) & =\sigma^{+}(P) \otimes \sigma^{+}(Q) & \sigma^{-}(M \oplus N) & =\sigma^{-}(N) \otimes \sigma^{-}(M) \\
\sigma^{+}(P \oslash M) & =\sigma^{+}(P) \otimes \sigma^{-}(M) & \sigma^{-}(P \backslash M) & =\sigma^{-}(M) \otimes \sigma^{+}(P) \\
\sigma^{+}(M \otimes P) & =\sigma^{-}(M) \otimes \sigma^{+}(P) & \sigma^{-}(M / P) & =\sigma^{+}(P) \otimes \sigma^{-}(M) \\
\sigma^{+}(\downarrow N) & =\neg \sigma^{-}(N) & \sigma^{-}(\uparrow P) & =\neg \sigma^{+}(P)
\end{aligned}
$$

The extended logical vocabulary is reflected in the definition of structures:

$$
\Pi, \Sigma::=P|(\Pi \bullet \Sigma)|(\Pi \bullet-\Sigma) \mid(\Sigma-\bullet \Pi)
$$


Finally, sequents, as before, are of the form $\Pi, \Sigma \vdash s$ or $\Pi \vdash s: N$, and made subject to the following inference rules:

$$
\begin{array}{ccc}
\frac{\Pi \vdash s: N}{p^{x} \vdash x: \bar{p}} A x & \frac{\Pi, P^{x} \vdash s}{\Pi \vdash \downarrow N^{x} \vdash(x s)} \downarrow \\
\frac{\Gamma, \Delta \vdash s}{\Delta, \Gamma \vdash s} d p^{1} & \frac{\Pi, P^{y} \bullet Q^{z} \vdash s}{\Pi, P \otimes Q^{x} \vdash \operatorname{case} x \text { of }\langle y, z\rangle s} \otimes \frac{\Pi \vdash s: M \quad \Sigma \vdash t: N}{\Sigma \bullet \Pi \vdash\langle s, t\rangle: M \oplus N} \oplus \\
\frac{\Pi \bullet \Sigma, \Upsilon \vdash s}{\overline{\Pi, \Sigma-\bullet \Upsilon \vdash s} d p^{2}} & \frac{\Pi, P^{y} \bullet-M^{\perp z} \vdash s}{\Pi, P \oslash M^{x} \vdash \text { case } x \text { of }\langle y, z\rangle s} \oslash \frac{\Pi \vdash s: M \quad \Sigma \vdash t: P^{\perp}}{\Pi \bullet-\Sigma \vdash\langle s, t\rangle: P \backslash M} \backslash \\
\frac{\Pi \bullet-\Sigma, \Upsilon \vdash s}{\Pi, \Sigma \bullet \Upsilon \vdash s} d p^{2} & \frac{\Pi, M^{\perp y}-\bullet P^{z} \vdash s}{\Pi, M \oslash P^{x} \vdash \text { case } x \text { of }\langle y, z\rangle s} \oslash \frac{\Pi \vdash s: M \quad \Sigma \vdash t: P^{\perp}}{\Sigma-\bullet \Pi \vdash\langle t, s\rangle: M / P}
\end{array}
$$

Completeness w.r.t. traditional (unpolarized) LG may again be established through use of phase spaces. Details are left to the reader's imagination.

A competing proposal for associating $\mathbf{L G}$ with a formal semantics is offered by Bernardi and Moortgat ([3]), who define dual call-by-name and call-by-value continuation-passing style translations into the implicational fragment of intuitionistic multiplicative linear logic. The lack of products in the target language results in semantic types often containing a larger number of double negations. While the terms used in lexical semantics thereby also grow in size, it does offer the grammar engineer more possibilities for fine-tuning his denotations.

\subsection{Normalization by Evaluation}

As a final remark, we point out the constructivity of $\S 4.1$ 's semantic completeness proof: rather than finding a countermodel for each unprovable sequent, we found a model wherein every truth translates into a proof. Explicating the algorithmic content underlying said proof, we might be able to show normalization for

$$
\mathbf{C N L}^{\text {pol }}+\frac{\Sigma, P^{y} \vdash t \quad \Pi, P^{\perp x} \vdash s}{\Pi, \Sigma \vdash(\lambda x s \lambda y t)} \text { Cut }
$$

In other words, rather than ignoring the term labeling and demonstrating closure under Cut for provability, like we did in $\S 4$, we might instead ascertain the latter property to hold of the proofs themselves as well. As a result, D 17 and T19. when composed with L.1. may then be understood as a double negation translation for (unpolarized) CNL. Such an argument, when involving the algorithmic content underlying a demonstration of model-theoretic completeness, makes essential use of a formalization of the constructive metalanguage, say Martin-Löf type theory or the Calculus of Constructions, as clearly argued in [4]. We leave these issues for future research.

Acknowledgements. This work has benefited from discussions with Michael Moortgat and Vincent van Oostrom, as well as from the comments of three anonymous referees. All remaining errors are my own. 


\section{References}

1. Andreoli, J.M.: Logic programming with focusing proofs in linear logic. Journal of Logic and Computation 2(3), 297-347 (1992)

2. Belnap, N.: Display logic. Journal of Philosophical Logic 11(4), 375-417 (1982)

3. Bernardi, R., Moortgat, M.: Continuation semantics for the Lambek-Grishin calculus. Information and Computation 208(5), 397-416 (2010)

4. Coquand, C.: From semantics to rules: a machine assisted analysis. In: Börger, E., Gurevich, Y., Meinke, K. (eds.) Computer Science Logic, 7th Workshop, CSL '93. pp. 91-105 (1993)

5. Girard, J.Y.: A new constructive logic: classical logic. Mathematical Structures in Computer Science 1(3), 255-296 (1991)

6. Girard, J.Y.: On the meaning of logical rules II: multiplicatives and additives. In: Foundation of Secure Computation. pp. 183-212. IOS Press (2000)

7. De Groote, P., Lamarche, F.: Classical non associative Lambek calculus. Studia Logica 71, 355-388 (2002)

8. Hendriks, H.: Studied flexibility. Categories and types in syntax and semantics. Ph.D. thesis, ILLC Amsterdam (1993)

9. Hepple, M.: Normal form theorem proving for the Lambek calculus. In: COLING. pp. $173-178$ (1990)

10. Herbelin, H., Lee, G.: Forcing-based cut-elimination for Gentzen-style intuitionistic sequent calculus. In: Ono, H., Kanazawa, M., de Queiroz, R.J.G.B. (eds.) WoLLIC'09, Tokyo, Japan. Proceedings. pp. 209-217 (2009)

11. Montague, R.: Universal grammar. Theoria 36(3), 373-398 (1970)

12. Moortgat, M.: Symmetric categorial grammar. Journal of Philosophical Logic 38(6), 681-710 (2009)

13. Okada, M.: A uniform semantic proof for cut-elimination and completeness of various first and higher order logics. Theoretical Computer Science 281(1-2), 471$498(2002)$

14. Prawitz, D.: Natural Deduction. Dover Publications (2006)

15. Wadler, P.: A taste of linear logic. In: Borzyszkowski, A., Sokolowski, S. (eds.) MFCS'93, Gdansk, Poland. Proceedings. pp. 185-210 (1993)

16. Y. Lafont, B.R., Streichter, T.: Continuation semantics or expressing implication by negation. Technical Report 93-21, University of Munich (1993)

17. Yetter, D.: Quantales and (noncommutative) linear logic. Journal of Symbolic Logic 55(1), 41-64 (1990)

18. Zeilberger, N.: The logical basis of evaluation order and pattern-Matching. Ph.D. thesis, Carnegie Mellon University (2009) 